Cahiers de recherches médiévales

Journal of medieval studies

5 | 1998

Le choix de la prose (XIIIe-XVe siècles)

\title{
« Nous chantons chansons diverses et si tirom diverses cordes »
}

L'esthétique de la dissonance dans le Tristan en prose

\section{Bénédicte Milland-Bove}

\section{(2) OpenEdition}

\section{Journals}

Édition électronique

URL : https://journals.openedition.org/crm/1402

DOI : $10.4000 / \mathrm{crm} .1402$

ISSN : 1955-2424

\section{Éditeur}

Honoré Champion

Édition imprimée

Date de publication : 30 octobre 1998

Pagination : 69-86

ISSN : 1272-9752

Référence électronique

Bénédicte Milland-Bove, « « Nous chantons chansons diverses et si tirom diverses cordes » », Cahiers de recherches médiévales [En ligne], 5 | 1998, mis en ligne le 01 octobre 2007, consulté le 15 décembre 2022. URL : http://journals.openedition.org/crm/1402 ; DOI : https://doi.org/10.4000/crm.1402

Ce document a été généré automatiquement le 15 décembre 2022.

Tous droits réservés 


\title{
« Nous chantons chansons diverses et si tirom diverses cordes »
}

\author{
L'esthétique de la dissonance dans le Tristan en prose
}

Bénédicte Milland-Bove

1 Par rapport à celle des romans qui l'ont précédée, la prose du Tristan frappe par son manque de couleur, par sa très grande prévisibilité. À force d'ériger en "coutume» fictionnelle et narrative, tout ce qui était auparavant présenté comme opérateur inédit de récits, à la pertinence et à l'occurrence unique, le style (en tant que phénomène global, incluant des effets sémantiques) du Tristan semble normé, voire normalisé. En même temps, des effets de rupture très forte fondent la réussite du roman.

Deux types de passage se détachent sur l'ordinaire de la narration. D'une part, on trouve des passages en "style soutenu "1, à fort investissement rhétorique, en prose comme les lettres ou les monologues lyriques, ou utilisant le démarcatif fort du passage au vers comme les «lais». De plus, des épisodes dialogués mettent en scène des personnages nouveaux dont le discours joue sur des décalages comiques et critique les poncifs idéalisants que le roman met par ailleurs très largement à contribution. Symboles de ces voix dissonantes, introduisant une tonalité nouvelle dans la prose arthurienne, la Demoiselle Médisante et Dinadan se révèlent, malgré les jugements contrastés de la narration à leur égard, étonnamment proches. Mais au fil du roman, on s'aperçoit que cette envoiseüre et les nouvelles valeurs qu'elle entraîne ne sont pas réservées à ces deux porte-parole. Tout chevalier, y compris Tristan, y compris le grave Lancelot, peut, à l'occasion, entrer dans le jeu. Le rôle du narrateur, intervenant sans cesse pour préciser ce qui doit être pris a certes et ce qui relève du gab, se révèle alors primordial pour assurer sans heurt la coexistence de ces discours hétérogènes.

3 Dans son désir de complétude, le Tristan en prose semble vouloir expérimenter les greffes les plus diverses sur sa trame narrative. Avec les monologues amoureux et les lais, il s'adjoint les ressources de la lyrique. Avec les interpolations de la Queste del saint Graal, il fait ressortir sa capacité à accueillir une écriture formellement assez proche mais à l'esprit et aux valeurs totalement différents. Dans cet article, on voudrait donc poursuivre l'exploration de ce jeu sur les dissonances, principe fondateur de 
l'esthétique du Tristan, en s'intéressant aux autres effets de rupture, ceux qui introduisent dans le roman en prose une tonalité plus familière et plus souriante ${ }^{2}$.

«Ha! demoisele, fait Mordrez, or oi merveilles de vos. Onques mes, se Diex me conseut, ne vi demoisele tant anuieuse com vos estes, qui onques ne cessez de dire vilenie a cest chevalier, et sanz deserte » (Édition R.L. Curtis t. III, p. $8^{3}$ ).

«Je ne trovai piecha mais cevalier ki si bien se seüst desfendre par paroles com vous faites» (I, p. 191).

«Il [Palamidés] n'oï onques mais chevalier [...] ki en tel guise parla d'amours com chis a fait» (IV, p. 156).

4 Par ces réflexions attribuées aux autres protagonistes du roman, l'auteur du Tristan attire l'attention du lecteur sur la singularité des discours qu'il met dans la bouche de Kahédin, la Demoiselle Médisante ou Dinadan. Voix dissonantes, ces personnages le sont certes par le contenu de leurs discours : la Demoiselle médit d'un jeune chevalier destiné, comme tout Bel Inconnu, à se métamorphoser en héros, Kahédin critique les us et coutumes du royaume de Logres, Dinadan dénonce les dogmes «pesants » de l'amour courtois et prétend les remplacer par un nouvel art d'aimer qui ressemble fort à de l'indifférence... Mais ces critiques, on l'a dit bien souvent, ne constituent pas une remise en question fondamentale de l'idéologie courtoise et chevaleresque. Folie inséparable du sens, - à moins que ce ne soit l'inverse -, "pro» insensé sans son " contra», les "dits plaisants » du Tristan en prose valent avant tout comme recherche littéraire, virtuosité stylistique source de comique, annexion des potentialités d'un autre registre, délaissé jusque-là par cette prose si sérieuse qui est celle des premiers cycles. Introduction d'une tonalité plus familière, détournement des discours orthodoxes, remise en cause des stéréotypes de langage, juxtaposition de registres différents, allusions explicites aux "genres comiques » : telles sont les marques propres de ces voix dissonantes.

5 Au récit des rivalités et des luttes chevaleresques, le Tristan en prose ajoute des dialogues où l'opposition verbale se substitue aux conflits physiques. A côté des bons combattants, il valorise donc les beaux parleurs et montre que la parole peut être une arme aussi redoutable que l'épée. Ainsi, ce n'est pas un hasard si, au tome IV, le harpeur qui interprète le Lai «Voir Disant» devant Marc, Helyot l'Envoisié, porte presque le même nom que le chef des Saxons qui vient d'attaquer la Cornouailles, Helyant. Des deux attaques, la plus redoutable n'est certainement pas celle que l'on croit... Dinadan se distingue par son excellence dans cette utilisation offensive de la parole et Tristan prend acte de cette nouvelle possibilité d'action :

«Je douteroie plus son sens que je ne feroie sa force » (IV, p. 348).

6 Au niveau le plus voyant, le personnage dissonant se distingue donc par son agressivité verbale, qui peut aller jusqu'aux insultes et aux malédictions. L'effet comique est d'autant plus fort qu'elles s'adressent à un personnage respecté de tous. Comme la Demoiselle Médisante qui provoque Brun dans l'espoir qu'il cessera de l'accompagner, Dinadan déclare violemment son désir de renoncer à la compagnie de Tristan :

«En non Dieu, danz chevaliers, de vostre compaingnie n'ai je cure. [...] A maufé

comant li un et l'autre» (éd. R. L. Curtis t. III, p. 11).

«Vostre compaingnie ait male aventure ! Je le vous laisse chi du tout !» (II, p. 122)

«Et quant nous aurom jousté, diable vous prende et vous et aus» (II, p. 131)

«Maudis soit il et confondus, et destruis soient tout chil ki le maintiennent » (II, p. 146). 
7 Plus encore qu'aux insultes directes, les personnages médisants recourent à l'ironie et à l'antiphrase pour humilier ou déstabiliser leurs adversaires. Ainsi, lorsque le chevalier à la Cotte Maltaillée, abattu par Palamidés, revient tout penaud vers la demoiselle, celle-ci l'accueille par ces mots :

«Or sus! frans chevaliers, or sus! Certes, bien l'avez fait. Or poez vos auques conoistre vostre boine chevalerie !» (éd. R.L. Curtis t. II, p. 237).

8 De même, Dinadan, ayant assisté à la chute de Tristan lors d'une joute contre Palamidés s'empresse de jeter de l'huile sur le feu.

De plus, la demoiselle partage avec Kahédin et Dinadan le don de dénoncer les habitudes et les stéréotypes de langage des chevaliers. Kahédin en Logres s'insurge contre l'absurdité des saluts qui président à toute rencontre entre chevaliers. Bien après sa disparition du roman, c'est Dinadan qui reprend ce rôle en stigmatisant les «Gardez vous de moi » et les provocations systématiques à la joute.

«Je truis si souvent u roiaume de Logres 'gardés vous de moi' c'a mon avis couvenroit bien a moi une garde ki ne feïst fors moi garder !» (V, p. 171).

Ces répliques sont donc l'occasion pour le prosateur de faire montre d'une autre virtuosité verbale, celle des jeux de mots utilisant la paronomase :

«Biaus ostes, se voirement me consaut Diex, vostre maisons n'est pas herberge, ains est tout droitement osteus, car il oste menu et souvent ses ostes» (II, p. 133).

11 De même, la demoiselle se plaît à prendre au pied de la lettre les formules rituelles qui ouvrent le discours des chevaliers. Comme l'explique C. Marchello-Nizia à propos de la locution "si m'aït Diex", ces expressions ont pour fonction de renforcer l'affirmation que le locuteur ajoute dans la suite de son énoncé et d'en gager la véracité sur cette valeur sûre qu'est la référence divine ${ }^{5}$. À deux reprises, la demoiselle répond par des sarcasmes aux déclarations confiantes de Brun ou du vieux chevalier père de Néronnéüs :

«Se Diex voloit, vos la [l'aventure] menriez a fin. Aussi deviendriez vos beste, se Diex voloit » (éd. R.L. Curtis t. II, p. 227).

«En non Dieu, fait la demoiselle [...] se Diex voloit, il feroit d'un mouton une jument ! [...] A ce ne devons nous pas entendre, car Diex ne demostre pas son pooir en chascun fet. » (éd. R.L. Curtis t. III, p. 24).

12 En détruisant la partie de l'affirmation qui postule la possibilité d'une intervention divine, la demoiselle annule du même coup, sans que son adversaire puisse répliquer, l'autre partie de son énoncé qui fait état d'une victoire possible. Dinadan oppose la même logique à Tristan, qui veut l'entraîner dans un combat inégal, et à Marc, quand ils doivent acquitter le passage d'un pont :

«Il m'est avis tout chertainnement que se Diex proprement m'i avoit mis, se m'i oublieroit il dou tout !» (II, p. 107).

Marc: «Par cele foi que je doi Dieu, je m'en deliverai bien, se Diex plaist. - En non Dieu, fait Dynadans, ce sai je bien. Se Diex voloit, il vous avroit ja mout tost cangié le cuer de Cornuaille que vous avés $u$ ventre a aussi grant cuer et a aussi hardi com a maintenant mesire Tristrans. Mais je sai bien qu'il ne s'en entremetra ja a ceste fois » (IV, p. 98).

Le personnage médisant feint de ne pas être au courant des habitudes de langage, il redonne aux expressions lexicalisées, figées, conventionnelles, leur sens littéral. S'attachant à un détail, il reprend à la lettre les propos de son interlocuteur pour donner une apparence de logique à ses railleries. Même s'il ne faut pas s'exagérer la 
portée de ces saillies, il est clair qu'elles s'attaquent au principe selon lequel Dieu, dans l'édifice médiéval de la parole, est le garant suprême de la vérité du langage ${ }^{6}$. Elles ouvrent ainsi la porte à une manipulation possible des énoncés.

Dinadan utilise à plus grande échelle la même méthode pour déconstruire le discours amoureux. Au tome IV ${ }^{7}$, pendant l'équipée de Marc en Logres, Palamidés, Dinadan et Marc se trouvent rassemblés fortuitement dans l'obscurité au bord d'une fontaine. Dinadan, après avoir écouté les lamentations amoureuses de Palamidés, se lance dans un véritable plaidoyer pour lui démontrer la folie de son obstination amoureuse. De manière significative, il commence par résumer le discours de Palamidés, pour mieux le détruire après cette reprise. À l'amour mortifère dont se plaint Palamidés, il oppose un amour insouciant, égoïste et teinté de misogynie. Mais surtout, il entreprend une démonstration dans laquelle il fait jouer de manière comique les métaphores amoureuses. Le rire naît tout d'abord du décalage entre les métaphores appelées (la métonymie classique du cœur de l'amant donné à sa dame) et l'apparat logique de la démonstration: les «ore donc» qui ponctuent son discours, les invitations à l'examen raisonnable qu'il adresse à Palamidés («Or gardom entre toi et moi a chestui point se ce puet estre vérité. Je te mousterrai par raison selonc ce qu'il m'est avis»). Ce raisonnement qui se veut logique, rationnel, contraste avec la manière peu rigoureuse dont est utilisé le discours amoureux. En effet, Dinadan insère dans un discours globalement métaphorique des décrochages qui rappellent les réalités physiques, feignent d'oublier le jeu métonymique et tirent le propos vers l'absurde et le burlesque.

"Onques voir sans mon cuer ne fui, anchois le sent souventes fois en mon pis batre et remuer » (IV, p. 154).

De même, après avoir fait le compte des amants d'Yseut, il rappelle :

«ele a tant de cuers en son pis qui n'est pas trop gros " (IV, p. 155).

Ce refus et cette feinte naïveté devant le langage imagé ne l'empêchent pas, dans une stratégie plus développée, de filer la métaphore. Iseut devient l'« ostel » qui reçoit tous ces cœurs et le lieu d'une lutte des cœurs dont Tristan, bien sûr, sortira victorieux. Poussé à bout, le discours amoureux est détourné, caricaturé et dénoncé dans son absurdité. Dinadan finit par une comparaison financière, qui vient signer ce détournement comique (bourgeois ?) de la parole amoureuse :

«Tu fais tout autresi com le fol povre home ki compere le sien petit avoir et melle avec l'avoir du riche home, et puis ne garde l'eure quant li grans avoirs du riche home a mengié son petit avoir» (IV, p. 155).

Comparaison surprenante dans le cadre du roman arthurien en prose où l'argent n'est pratiquement jamais mentionné.

C'est donc par une série de juxtapositions incongrues, qui insèrent dans un discours apparemment classique mais poussé jusqu'aux limites de la caricature des notations étonnantes, que procèdent les voix dissonantes du Tristan en prose. Dans ce détournement des formes, Dinadan ira encore plus loin. Le narrateur lui attribue en effet le lai satirique composé en réponse à l'agression verbale de Marc contre Guenièvre. Certes, c'est bien avec Marc que le roman a inauguré la série des messages d'insultes échangés par les héros. Mais le travail de Dinadan se présente expressément comme la reprise du schéma rimique et de la mélodie du «Lai Mortel» de Tristan. Il y a donc bien volonté de détourner le discours amoureux, et Dinadan se justifie de son invention en ces termes : 
«Il [Marc] est mauvais et anieus et vilains, et letres anieuses et vilainnes li doit on mander. [...] Li autre ki a celui tans avoient ja trouvé maint lay avoient canté et parlé de bonté et de courtoisie, pour ce k'il savoient chertainnement que chil estoient courtois et boin de qui il cantoient. Ore donc, pour ce k'il set vraiement, et tous li mondes le set bien autresi, que li rois March est li plus caitis rois et li plus noiiens de tot le monde, li plus vieus et li plus faillis, se il fait lay de sa vergoigne et li conte sa mauvaistié, tous li mondes en avra joie» (IV, p. 278).

19 Tout se passe comme si Dinadan, utilisateur réaliste du langage, prenait acte de sa face sombre, ordurière, «aigre » (c'est ainsi que Tristan qualifie le lai ${ }^{8}$ ). Avec lui, le roman en prose revendique son droit à insérer en son sein des écrits portant la marque d'une habileté rhétorique différente de celle du beau langage, mais qui n'est pas sans potentialités... et qui se justifie par la théorie de l'adéquation au personnage ${ }^{9}$. La qualité rhétorique du lai est soulignée par la narration qui précise que le lai est «bel et bien dit $»^{10}$ et qui évoque le plaisir qu'il suscitera chez les auditeurs. Cette mise en scène de l'écriture du lai de Dinadan est donc également une manière de justifier a posteriori l'insertion de la lettre en forme de lai du roi de Cornouailles, d'ailleurs beaucoup plus réussie et beaucoup plus riche en insultes pittoresques que celle de Dinadan... Ces «viles paroles» attribuées à Marc sont aussi recherchées, aussi travaillées que les autres registres, et font sans doute, dans l'esprit du prosateur, partie des «beaux dits, riches et plaisants $» .$.

20 Tous ces discours jouent donc de la parodie de formes que le Tristan lui-même s'est appliqué à promouvoir et à ériger en règle : c'est bien lui qui a inventé l'obligation de la joute pour les chevaliers errants du royaume de Logres et ces saluts en forme de défis, c'est bien lui qui reprend à son compte les métaphores courtoises, qui les utilise de manière littérale dans les lais et les lettres que s'envoient les personnages (par exemple, lorsque Tristan, après son mariage avec Iseut aux Blanches Mains, écrit à Lancelot pour lui demander conseil, l'image du «cœur séparé du corps ", redoublée concrètement par les considérations sur la distance géographique est abondamment exploitée $\left.{ }^{11}\right) \ldots$

21 Les voix dissonantes ont aussi en commun d'établir des analogies entre des registres de représentation très différents, créant ainsi des effets d'incongruité. On a vu que dans ses répliques stigmatisant la confiance démesurée des chevaliers, la Demoiselle Médisante utilisait des références au règne animal et dénonçait ainsi les habitudes de langage des chevaliers. Cette convocation cocasse des comparaisons animales est récurrente dans le roman. Ainsi, lorsque Dinadan cherche à se venger de Tristan et se moque de lui aux vêpres du tournoi du Château des Pucelles, il exprime de manière inattendue le défi qu'il lui lance :

«se vous estiés uns cevaus, je vous quideroie a celui point la keue nouer tout legierement» (II, p. 240).

Si le sens de cette remarque est clair (Dinadan se promet de voir Tristan vaincu, lassé et humilié avant la fin du tournoi), son origine l'est beaucoup moins. En effet, si l'on en croit le dictionnaire de Godrefroy, il existe plusieurs locutions intégrant le terme "queue » et signifiant "tromper", "jouer un tour pendard ": "faire la coe ", "faire le no en la coe »... Mais la référence au cheval n'est plus perceptible dans aucun des textes cités. Or, ici, Dinadan concrétise la locution en précisant «se vous estiés uns cevaus". Est-ce la trace d'une pratique consistant à attacher entre elles les montures des chevaliers vaincus pour éviter leur fuite, précaution prise par un nain félon dans Le 
Chevalier au Lion (il les a «coe a coe noëz $»^{12}$ ) ? Dans le Tristan, le rapport entre le cheval et son cavalier n'est plus seulement métonymique, mais il est poussé jusqu'à la personnification. Le Dictionnaire des symboles ${ }^{13}$ précise que la queue, et particulièrement celle du cheval, représente souvent le lieu de concentration de l'énergie et des forces vitales. D'où peut-être toutes ces locutions, que le Roman de Renart, lorsque Renart s'en prend à la cour pour échapper à Noble et attache pendant leur sommeil tous les animaux par la queue, se serait amusé à mettre en images ${ }^{14}$. Quoi qu'il en soit, il est remarquable que ce tour familier, mis dans la bouche de Dinadan, soit précisé par un détail concret qui le «re-littéralise » et le rend d'autant plus incongru. L'impertinence de cette image se retrouve dans d'autres saillies de Dinadan, faisant référence à des proverbes, ou, dans le deuxième exemple, au genre des récits animaliers :

«Vous veés souvent avenir que li leus n'est pas si grans que on le crie ne li

chevaliers ne sont souventes fois pas si preu com on raconte» (II, p. 239).

« et ce vous fist obeïssant plus que li leu ki pris est au piege » (V, p. 221).

L'allusion au registre de la fable animalière est encore plus nette dans d'autres passages du roman. Dinadan, à plusieurs reprises, réécrit sur le mode comique la rivalité entre Tristan et Palamidés. Éternels rivaux, les deux amants d'Iseut oscillent entre jalousie, rancœur, colère, admiration et estime mutuelles. Le roman raconte les hauts et les bas de cette "inimitié intime", alternant hostilités, humiliations, rêves de vengeance de Palamidés et actes de générosité des deux chevaliers, qui cohabiteront même un moment à la Joyeuse Garde. Le prosateur se garde bien de trancher dans un sens définitif, ne voulant pas tarir avec cette rivalité une source de récits si féconde.

Par jeu, Dinadan ne manque jamais une occasion d'attiser la rivalité des deux frères ennemis et se plait à dévoiler les attitudes ambiguës sous la belle façade courtoise des chevaliers. Après le tournoi du château des Pucelles, les trois chevaliers sont retenus en prison par Daras, dont Tristan a tué le fils. Dinadan révèle à Palamidés l'identité de son compagnon de captivité. Procédant par questions / réponses dans une sorte de dialogue socratique où Palamidés tire de lui-même les conclusions qui s'imposent, il élabore une petite fable à laquelle les deux protagonistes sont invités à s'identifier.

«Palamidés, fait il, se Dex vos saut, veïstes vos onques en un lieu metre ensamble deus bestes qui naturaulement s'entrehaïssent, ki longement se tenissent en pais?» (III, p. 189).

Que se passerait-il si l'on mettait ensemble un loup et une brebis? Le dénouement attendu est repoussé sitôt invoqué : il pourrait bien advenir que le loup soit mangé par la brebis...

Par ce double avertissement, qui vise Palamidés aussi bien que Tristan, Dinadan souligne le sel de la situation imaginée par le prosateur, son côté incongru et son issue imprévue (la réconciliation des deux rivaux). C'est avec une autre référence intertextuelle qu'il souligne le caractère provisoire de cette trêve :

"Ja Dieu ne plache que la vostre amour puisse mais tant durer com fist l'amour de Galehaut et de Lanselot du Lac, et non fera ele sans doute, che sai je tout vraiement!» (III, p. 191).

Le rôle de Dinadan est d'élargir au plus vaste ces possibles narratifs, d'évoquer d'autres images, d'autres registres... Grâce à ce personnage, le prosateur souligne sa différence avec le Lancelot, fait un clin d'oeil aux Isopets... La convocation de ces autres récits est une manière souriante d'enrichir les potentialités et les résonances du roman. D'une part, la comparaison animale a pour fonction de révéler que malgré leurs déclarations 
belliqueuses, les chevaliers n'ont guère envie de combattre. Palamidés surtout est saisi d'un mouvement de panique qui se transforme en un immense soulagement lorsque Tristan rappelle sagement (ou habilement?) qu'il y a un temps pour tout, et que les prisonniers feraient mieux d'unir leurs forces pour supporter leur captivité. Avec cette image partiellement dévalorisante, le roman semble également évoquer la possibilité d'une autre scène dégradante pour les chevaliers: Dinadan suggère de manière provocatrice le plaisir qu'il ressentirait à voir lutter à mains nues (« comme deux bêtes enragées »), les deux rivaux dans leur prison. Mais ces ouvertures sont aussitôt refermées, et le Tristan en prose met en valeur la solution courtoise qu'il apporte au conflit. La convocation de l'autre couple révèle qu'il y a peut-être surtout, entre Tristan et Palamidés, un rapport de fascination. Elle livre aussi sans doute un secret de fabrication: la volonté de créer un duo chevaleresque capable de rivaliser, en le renouvelant, avec celui formé par Lancelot et Galehaut dans le Lancelot.

Ces procédés seront repris plus loin dans le roman. Découvrant avec surprise le compagnonnage de Tristan et de Palamidés pendant une partie de la quête du Graal, Dinadan convoque explicitement le Roman de Renart, auquel on ne pouvait s'empêcher de penser dans l'épisode précédent. Dinadan exprime sa surprise par une série de comparaisons. Tristan et Palamidés ne peuvent pas plus cohabiter que la clarté et l'obscurité, ce sont deux principes contraires qui ne sauraient s'accorder et il conclut :

« et se concorde est entr'aus par aucun pecié, Renars et Ysengrins sont frere et bien voellans et cier ami ! » (VI, p. 354).

29 Là encore, la comparaison est à la fois appelée pour les analogies qu'elle suscite, les échos qu'elle éveille et en même temps elle est refusée par le texte puisque le comportement des deux héros s'est justement révélé différent de celui des deux animaux. Elle n'en ouvre pas moins des prolongements évocateurs à l'histoire des chevaliers. Dans le Tristan, ce n'est pas la première allusion au Roman de Renart. Au tome IV, à plusieurs reprises, la cour arthurienne avait mis en garde Tristan contre les agissements de son oncle, le roi Renart ${ }^{15}$. Ces références renardiennes, qui demeurent assez vagues et générales, autorisent cependant à voir dans ces passages "comiques» un procédé conscient d'annexion des ressources d'un autre registre, d'une autre matière.

«Nulle parole n'est estable

Verité est tornez a fable $»^{16}$

Ces lignes souvent commentées du Roman de Renart pourraient fort bien convenir au Tristan en prose, qui, par l'orchestration des dissonances, semble lui aussi remettre en cause la conception "symbolique $»^{17}$ d'un langage renvoyant à une signification stable, fondée sur un ordre de valeur indiscutable. Cependant, par rapport au Roman de Renart, dont la figure majeure est l'inversion, la marque propre du Tristan est, non le renversement, mais l'échange. Le plan des valeurs n'est pas bouleversé, le monde n'est pas bestourné... Mais l'image de Renart peut être convoquée à la fois pour Tristan, le meilleur chevalier du monde, et pour son oncle, le roi le plus vil. De même, on le verra, des termes antonymes comme folie et sagesse peuvent désigner les mêmes attitudes face à la prouesse et à l'amour... L'instabilité du signifiant ne remet pas en cause le signifié, elle se contente de désigner l'arbitraire du langage et la marge de jeu qui est offerte à l'écrivain. Le Tristan en prose exalte le pouvoir de la fiction de faire tenir ensemble les discours les plus différents, les plus contradictoires, sans qu'ils s'annulent. 
31 L'analogie existant entre le personnage de Renart et l'écriture de la renardie a souvent été soulignée ${ }^{18}$. De même, les voix de Dinadan et du narrateur tendent parfois à se confondre, parce que Dinadan est le joueur par excellence, porteur de ce discours autre, parallèle, mais sans intention subversive, que se plaît à insérer le prosateur. Cependant, Dinadan n'est pas le porte-parole de l'auteur. La disqualification de la Demoiselle Médisante est bien la preuve que le discours dissonant ne trouve pas sa justification en lui-même, mais qu'il n'est là que pour la distance ludique qu'il introduit par rapport au discours « orthodoxe».

32 C'est parce qu'elle n'obéit pas à ces règles implicites, parce qu'elle veut appliquer sérieusement le registre comique que la Demoiselle Médisante, jugée de plus en plus négativement au fil des aventures, finit par disparaître à tout jamais du roman. En effet, ni ses motivations initiales (éviter à un jeune chevalier de se faire tuer), ni la teneur de son discours (à peu près la même que celle des remarques de Kahédin et Dinadan) ne justifient en eux-mêmes son éviction. Seul l'esprit de ses propos est tout différent.

33 Tout se passe donc comme si seul un chevalier pouvait se permettre, de l'intérieur, une parole critique. Un épisode du tome II entérine en quelque sorte cette passation de pouvoir entre une autre incarnation de la Demoiselle Médisante et Dinadan ${ }^{19}$. La demoiselle, qui a vu Palamidés tomber dans une rivière et a échangé avec lui quelques propos peu amènes, le considère comme un couard. Aussi est-elle toute contente d'entendre Dinadan se moquer des rodomontades de Palamidés adressées à un Tristan qu'il croit loin. La demoiselle s'empresse de renchérir, mais Dinadan ne lui laisse pas très longtemps ce plaisir. Le chevalier fait taire la demoiselle, personnage impossible (parce que trop dangereux ?) et qui ne réapparaîtra plus dans le roman. La dérision doit être maniée à bon escient, par un personnage autorisé, c'est-à-dire par un chevalier conscient de la part de jeu dans ses propos, capable de laisser la place au discours inverse.

34 Le rôle de la narration est donc primordial: sans cesse, elle oriente le jugement du lecteur sur ces discours peu orthodoxes. Elle accompagne en effet les paroles des personnages de «didascalies » qui précisent le ton, l'esprit dans lequel est prononcé le discours, ton que ne saisissent pas forcément les interlocuteurs du personnage. Ainsi, lorsque Dinadan se moque de Tristan désarçonné dans une joute malheureuse contre Palamidés, le narrateur précise :

«il [Tristan] quide tout certainnement que Dynadans li die a certes les paroles k'il li disoit, mais non faisoit il sans doute: il li disoit par gieu et par envoiseüre» (II, p. 240).

Ces déclarations ne sont pas une manière d'affaiblir les discours dissonants, de leur ôter leur portée. Il s'agit de la revendication de cette distance qui est la marque propre de l'écriture du Tristan en prose. Ces voix dissonantes, loin d'être annulées par les précisions sur leur dimension ludique, n'annulent pas non plus les paroles qu'elles ont en face d'elles. Les discours opposés ont au contraire pour rôle de se mettre mutuellement en valeur, et de souligner la prouesse de l'écrivain qui réussit à faire coexister ces discours antagonistes.

Cela est très clair dans les épisodes où apparaissent ensemble Tristan et Dinadan. Au tome $\mathrm{II}^{20}$, un épisode manque mettre fin à l'amitié entre les deux compagnons. Tristan décide de lutter contre les trente chevaliers postés par Morgane en embuscade contre Lancelot. Dinadan, qui avait entendu trois au lieu de trente, refuse, lorsqu'il apprend le nombre exact des chevaliers, de tenter l'aventure. Il s'ensuit une joute verbale entre les 
deux chevaliers, où Dinadan reproche à Tristan sa «rage et forsenerie » et où Tristan, de son côté, taxe son compagnon de couardise. Dinadan lui-même souligne la différence qui existe entre lui et Tristan:

«Ensi sommes nous discordant entre moi et vous, car vous seriés hardis de nul

besoins, la u je seroie couars» (II, p. 112).

Comment se débrouiller avec deux personnages qui ne sont pas de même nature, qui n'obéissent pas à la même logique... et ne tiennent pas le même langage ? Pendant que Tristan se lance sans hésiter dans l'aventure, avec comme seule crainte celle d'arriver sur les lieux de l'embuscade après Lancelot et d'être ainsi privé de son exploit, Dinadan de son côté, adepte du proverbe «Bone fuie si vaut mout » qu'il développe dans une argumentation logique, préférerait rebrousser chemin. Ses remarques attestent son incompréhension à l'égard de la dimension héroïque représentée par Tristan :

«Sont ce donc bestes a departir? Nenil [...], ce ne sont mie bestes, ains sont cevalier tout armé » (II, p. 108).

Visiblement, Dinadan préfèrerait être le compagnon d'un Don Quichotte poursuivant des moutons, plutôt que celui de Tristan! La narration ne donne tort ni à l'un ni à l'autre: un vieux chevalier rencontré sur la route avertit Tristan du danger avec des incitations à la prudence qui redoublent celles de Dinadan. En même temps, l'issue du combat montre l'exceptionnelle valeur de l'amant d'Yseut, et justifie les hyperboles employées par le chef des trente chevaliers dans son rapport à Morgane :

«Dame, il n'est mis cevaliers, ains est destrusion de gent » (II, p. 119).

Cet épisode important, dans lequel le prosateur semble relever un défi d'écriture, marque clairement une volonté de se situer par rapport à l'univers textuel de son grand modèle, le Lancelot en prose. Ne donne-t-il pas à Tristan l'aventure préparée pour Lancelot? Tristan, comme Lancelot à la Douloureuse Garde, ne possède-t-il pas son écu "magique ", qui "vait ausi com doublant [sa] force " ${ }^{21}$ ? Mais la magie est ici celle de l'amour, puisque l'écu est un cadeau d'Iseut. Quand il apprend cette vertu, Dinadan qui, par crainte d'être pris pour Lancelot, avait demandé à Tristan de lui prêter son écu de Cornouailles, décide finalement de ne pas opérer la substitution. Dans ce passage où la thématique de l'échange est omniprésente, où les écus représentent métonymiquement l'individualité propre de celui qui les porte, n'est-ce pas aussi l'identité du texte qui se joue? C'est l'introduction du personnage dissonant, c'est cette juxtaposition signifiante de Dinadan et Tristan qui souligne le changement d'univers textuel, qui donne sa marque, son style propre au Tristan au prose.

En dernier ressort, que penser des refus de combattre de Dinadan, sinon les prendre $a$ gas comme l'intéressé ? Dinadan les présente comme une preuve de sagesse, mais dans certains épisodes, son attitude est montrée sous un jour plutôt défavorable. C'est le cas par exemple au tome III. Lors d'une étape, Dinadan et Keu entendent un chevalier Vermeil, sans doute fils de Galehaut, mettre en doute la courtoisie de Guenièvre. Dinadan refuse de jouter à la suite de Keu pour venger l'insulte et admet sereinement son déshonneur:

«Chevaliers ne porroie je estre encontre vous, car trop estes plus fors de moi et mieudres» (III, p. 266).

41 La narration passe à autre chose sans donner tort ni raison à Dinadan. A plusieurs reprises, Dinadan revendique une prouesse à sa mesure. Devant Iseut qui lui demande de combattre pour elle contre trois chevaliers, il déclare qu'il n'est ni un ogre, ni un 
géant $^{22}$. Par ce refus des hyperboles, Dinadan exprime sa non-appartenance au personnel traditionnel du roman arthurien et signale l'intégration au sein du roman en prose d'un registre plus «médiocre ».

De plus, l'échange des rôles, la réécriture qui reprend la même matrice formelle en modifiant les protagonistes, viennent sans cesse redistribuer les cartes. Ainsi, l'attitude de Marc face aux six chevaliers que Dinadan lui demande, par jeu, d'affronter ${ }^{23}$ rappelle celle de l'ami de Tristan face aux trente chevaliers de Morgane. Dinadan a inventé de toutes pièces cette hostilité imaginaire entre lui et six chevaliers de la Table Ronde, qui sont en fait tranquillement en train de déjeuner un peu plus loin. Dans les mêmes termes que jadis Tristan, il exhorte Marc à ne pas tourner bride et à venir hardiment au combat, et c'est Marc qui invoque la raison et le déséquilibre des forces pour lui fausser compagnie.

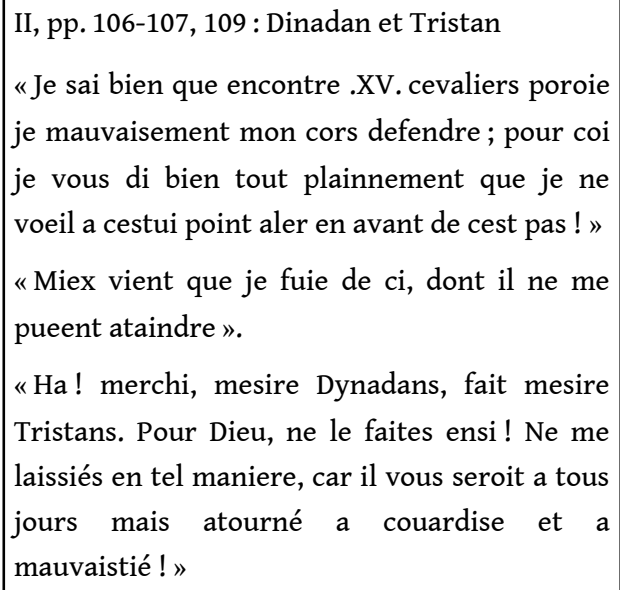

IV, p. 118 : Marc et Dinadan

«Chis meschiés est trop perilleus que deus chevaliers encontre .VI. [...] pour che vous di je tout plainnement que je vous laisse en chestui fait. Je ne vous tenroie compaignie en nule maniere du monde. »

«Mieus vaut que on se garde de loing que on atende de trop pres.

«Ha, sire ! fait Dynadans, pour Dieu merchi, ne le faites ensi, mais regardés a vostre honour ». absolu, tout peut sans cesse être remis en jeu. Les mêmes discours, les mêmes postures peuvent être adoptés par des personnages bien différents et prendre ainsi des valeurs contradictoires.

Enfin, Dinadan lui-même peut, à l'occasion, oublier ses principes: se mettre en aventure d'une joute, et même tomber amoureux d'une demoiselle au point de se battre avec Gaheriet "comme deus bestes esragies $»^{24}$. Au tome IV, lors d'une discussion sur l'amour avec Tristan et Espynogre, le narrateur nous présente un Dinadan «esragiés et forsenés ", prêt à se battre en invoquant la coutume de Logres pour défendre le bienfondé de son opinion, se répandant en insultes devant les refus de son interlocuteur:

«Dinadans se courece mout fort de ces paroles que mesire Tristans li avoit disoit par envoiseüre, mais Dinadans quidoit mout bien que ce fust a certes » (V, p. 108).

C'est Gaheriet qui lui rappelle les règles du jeu :

«il avient souventes fois que maint cevalier dient par lour soulas et par lour envoiseüres teus paroles que on ne trouveroit mie en lour fais se ce venoit au grant besoig [...]. Li cevaliers vos disoit par aventure par son gieu ce que vous entendés a certes» $(\mathrm{V}, \mathrm{p} .147)$.

Le roman souligne ainsi les mécanismes ludiques de son écriture. Le langage, et le roman tout entier, sont conçus comme un jeu qui se prête à toutes les variations, 
reprises, transformations, échanges. Si le texte peut sembler parfois contradictoire, il s'agit en fait d'une volonté de faire contraster deux attitudes, de prendre le contre-pied du schéma attendu, de placer un personnage dans une situation inédite. L'utilisation étendue que fait le texte du vocabulaire de la sagesse et de la folie contribue également à donner l'impression d'une reprise et d'un échange perpétuels, vertigineux, entre les termes et les jugements de valeur dont ils sont porteurs. Dinadan en est l'utilisateur le plus virtuose. Il les insère dans un discours sentencieux qui n'est rien d'autre que la reprise et l'amplification d'une autre forme littéraire, celle des proverbes.

Le goût du Tristan pour les proverbes est une nouveauté pour la prose. En effet, on trouve très peu de proverbes dans le Lancelot ou le Perlesvaus, et il n'y a donc pas de proverbes dans les parties du Tristan reprises au Lancelot et à la Queste del saint Graal. Cette large utilisation du matériau proverbial s'explique peut-être par les caractéristiques mêmes du proverbe, qui «met en champ deux énoncés contradictoires $»^{25}$, réunit dans la structure unifiante thème/prédicat des éléments n'appartenant pas au même paradigme. De plus, comme le souligne P. Ménard à propose de certains d'entre eux, particulièrement plaisants, le proverbe «illustre une situation romanesque avec des éléments empruntés à un autre registre. Il opère une transposition, et parfois introduit une sorte de plaisante dissonance ${ }^{26}$. Le Tristan renoue ainsi avec les pratiques des romans en vers, notamment ceux de Chrétien de Troyes. Parmi ces proverbes, nombreux sont ceux qui déclinent les termes de «folie » et «sagesse » et brouillent le mécanisme des associations axiologiques.

Ainsi, l'histoire de la Demoiselle Médisante et du chevalier à la Cotte Maltaillée peut se lire comme l'«exploitation ${ }^{27}$ de deux proverbes. Selon le jeune chevalier, qui se fait l'expression de l'idéologie chevaleresque par excellence et utilise un proverbe très fréquent dans les romans en vers ${ }^{28}$, «Mieus vaut morir a honneur que vivre a honte». La Demoiselle Médisante en prend bien sûr le contre-pied:

"Coment, ce dit la demoisele, a il donc en vos si peu de sens et de reson que vous volez mieuz morir que vivre?» (éd. R. L. Curtis t. II p. 236).

Elle dénonce le manque de bon sens du chevalier :

«Sire chevaliers, ensi va des choses dou monde que quant li hons de povre sens troeve conseil, il ne le veust croire». (éd. R. L. Curtis t. II, p. 228)

«Toz jors, fait la demoisele, se va li fox reconfortant de sa folie » (éd. R. L. Curtis t. II, p. 238).

Ainsi, pour la demoiselle, l'attitude du jeune chevalier relève d'une témérité excessive qu'elle condamne. Cependant, au fil des aventures, Brun prouve sa valeur. La demoiselle n'en modifie pas pour autant son attitude à l'égard du chevalier, et son jugement défavorable se trouve désormais en contradiction avec les faits.

51 Dans la bouche de Dinadan, le terme de «folie » devient aussi synonyme de "prouesse », mais il en arrive presque à perdre sa valeur péjorative. Quand Dinadan dit «folie » pour parler de la vaillance des chevaliers, cela ne bouleverse nullement la hiérarchie des valeurs. Ainsi, lors d'un séjour de Dinadan à la cour d'Arthur, la conversation est mise sur la prouesse comparée de Palamidés et Lamorat. Avant de se déclarer en faveur de Lamorat, Dinadan précise :

«je sai bien tout chertainnement que pour grant sen trouver ne m'acorderoie je ne a l'un ne a l'autre. Mais pour folie si feroie» (IV, p. 204). 
cette logique, Tristan est le «maitre des fous " ${ }^{29}$. Il rejoint donc paradoxalement la figure de Daguenet, fou véritable mais que sa folie empêche de prendre conscience du danger :

«Il est si fox k'il ne refuseroit l'encontre de nul chevalier du monde » (IV, p. 123).

Mais le lecteur ne doit-il pas être capable de distinguer entre l'emploi métaphorique, pour rire, du terme par Dinadan et son sens propre? meilleurs chevaliers en matière de prouesse se double d'une folie amoureuse qui est dénoncée plus vigoureusement encore par Dinadan. Ainsi, paradoxalement, par le biais du terme "folie», l'alliance entre amour et prouesse se trouve confirmée dans l'idiolecte même du personnage. Au tome $\mathrm{V}$, devant l'échec de ses tentatives pour montrer la supériorité des chevaliers qui «n'aiment pas d'amour» sur les autres, il se plaint d'être tombé dans une « histoire de fous»:

«Et tele est sans doute l'aventure des faus que ki entre faus s'embat plus est faus que devant, pour k'il demeurt longement entr'aus! Quant aventure m'aporta entre vous, je estoie assé faus, bien le connois, mais or sui je plus que faus, et ma folie m'a fait honte a cestui point. Pour ce dist trop bien ki le dist : 'de fol te garde, si vivras em pais!'» (V, p. 111).

Le narrateur lui-même, à certains moments, semble adopter le langage et les valeurs de Dinadan. L'expression «entrer en la folie» peut par exemple devenir synonyme de «jouter»:

«Li rois entre en la folie et prent son escu et son glaive pour jouster » (IV, p. 91).

«Et pour ce k'il [Dinadan] se sentoit adonc auques bien ne n'estoit pas mout traveilliés, emprent il sour soi la folie; car on voit souvent avenir que li plus sages vait foloiant aucunes fois ». (IV, p. 108) $)^{30}$.

Cet exemple est la seule occurrence dans tout le Tristan en prose d'un proverbe qui ne soit pas attribué à l'un des personnages mais appartienne au discours propre du narrateur, dans une intervention lourde où le « je » s'affirme avec vigueur :

«cheste parole ai je ore dite pour Dynadant, ki bien peüst celui pont passer tout franchement, se il vausist, sans jouste et sans bataille avoir » (IV, p. 108).

La voix du narrateur ne se rapproche donc véritablement de celle de Dinadan que dans cet instant où il souligne les contradictions du personnage! Malgré tout, il est significatif qu'il double la hiérarchie fondée sur la prouesse par une autre fondée sur l'envoiseüre. Ainsi, lorsqu'au tome IV, il fait intervenir Girfflet, il précise dans sa présentation:

«ki compains estoit de la Table Reonde, et cevaliers durement envoisiés, et boin gabeour avoit en lui» (IV, p. 123).

De même, le discours désabusé de Dinadan sur l'amour entre en coïncidence avec des passages où la narration semble se plaire à présenter une autre image de la femme, à rappeler la possibilité d'un discours "autre». La teinte proverbiale de ces discours dissonants est également très nette. Par exemple, dans l'épisode de Dinas le sénéchal et de la dame volage, on trouve des variations sur deux séries de proverbes. L'un dénonce le cœur volage des femmes:

«Miex vaut la nature des chiens, et est plus gentiex et plus franche, plus loiaus et plus enterine, que la nature des femes, ki sont mauveses » (III, p. 176) 
et l'autre glose la distance entre paroles et actions : «Entre faire et dire a moult». Ces deux proverbes forment la base des discours dissonants sur l'amour: Dinadan, confronté aux exigences d'Iseut au tome $\mathrm{V}$, les reprend à son compte :

«[...] cuers de feme est tout ausi com la venvole: or aimme, or het, or pleure, or cante» (V, p. 129).

À la reine, qui pour l'éprouver, lui demande de la défendre dans une querelle imaginaire contre trois frères, Dinadan répond, indigné :

«or saciés certainnement que li requerres est legiers, il ne vous grieve se poi non a

requerre, mais li fais en est mout greveus !» (V, p. 131).

61

Cependant, il faut dans tous les cas rétablir la distance ludique du gab. Liés à des personnages emblématiques qui fonctionnent comme des signaux textuels, embrayeurs de la tonalité comique, ces passages sont sous le signe de l'envoiseüre. Certains commentaires du narrateur, qui mêlent le vocabulaire de la sagesse et de la plaisanterie, sont là pour le rappeler, comme par exemple lorsque Dinadan tourne en dérision la pratique des joutes "par amour»:

"Quant li chevaliers ot Dynadant parler si sagement, il ne s'aperchoit mie adonc que Dynadans li die ces paroles par gas, ains quide bien k'il li die tout a certes ». (IV p. 199).

De même, Tristan et Yseut, représentants par excellence de l'«autre » discours sur l'amour, sont capables d'apprécier les propos de Dinadan :

«Dynadans set assés plus que je ne quidoie et que vous ne deïssiés. Onques mais ne vi cevalier de si sages paroles ne de si boines!» (V, p. 135).

Contrairement à la Demoiselle Médisante, qui excluait la légitimité du discours inverse et visait à la destruction de toute contradiction, le Tristan veut harmoniser et faire jouer ensemble ces différents registres. À tous les niveaux, il pratique une esthétique du contraste qui habitue le lecteur aux juxtapositions les plus hardies. Dans cette perspective, la forme-sens qui définirait sans doute le mieux le Tristan en prose est celle du jeu-parti ${ }^{31}$. Ainsi, dans la discussion sur l'amour qui oppose Palamidés et Dinadan, celui-ci invite explicitement son interlocuteur à défendre son opinion : "Or deffent ta partie au mieus que tu porras ». Déçu par le silence de son adversaire, il insiste : «Por coi ne deffens tu ta partie?» S'étant aperçus de la présence de Marc, les deux chevaliers lui demandent de prendre position dans le débat:

«Dites moi a laquele partie de nous deus vous vous tenés orendroit» (IV, p. 158).

concilie les deux opinions et renvoie ainsi le lecteur au point de départ : l'attitude de Dinadan est, certes, plus raisonnable, mais peut-on lutter contre la force d'amour? des tonalités contraires, mais au sein même des discours sérieux : ainsi, la plupart des monologues lyriques offrent une structure en contraste ${ }^{32}$, avec d'abord une condamnation, puis un éloge du pouvoir de l'amour.

"Nous chantons chansons diverses et si tirom diverses cordes $»^{33}$, déclare Dinadan à Tristan lors de leur grand débat sur l'amour. On pourrait prendre cette expression dans un sens métatextuel pour caractériser l'esthétique du Tristan en prose. Comment le roman réussit-il à orchestrer cette diversité des registres, cette juxtaposition des tonalités et des univers textuels les plus éloignés? Comment, par exemple, insérer les mésaventures amoureuses de Dinadan, qui a accepté de prendre en conduit une 
demoiselle, au beau milieu des interpolations de la Queste $\mathrm{du} \mathrm{Graal}^{34}$ ? Cette juxtaposition maximale est possible, car tout au long de l'œuvre, le prosateur n'a cessé de jouer des effets de contraste entre un "tissu narratif simple», et d'autres passages marqués par une recherche stylistique (que cette recherche produise un «style soutenu » ou un style " comique ").

De façon assez remarquable, le Tristan en prose est sans doute le premier à imposer l'idée d'une norme courtoise, norme qui se traduit à la fois sur le plan stylistique et sur le plan fictionnel, par la référence à des coutumes. En effet, non seulement le texte fait un large usage des motifs narratifs et des formules empruntés aux romans qui l'ont précédé, mais il les érige en coutumes fictionnelles que certains personnages du roman auront à cœur de respecter. Ainsi, il reprend la séquence traditionnelle qui enchaîne la rencontre entre deux chevaliers, l'échange de saluts ou de défis, la joute, le combat à l'épée, la reconnaissance et l'arrêt du combat. Il redouble au plan de la diégèse ce déroulement codé: les chevaliers errants du royaume de Logres sont obligés de se provoquer quand ils se rencontrent, un compagnon de la Table Ronde n'a pas le droit d'en combattre un autre, etc.

Mais dans cet univers où tout est codifié, la norme n'est là que pour être transgressée, elle ouvre la voie à des séquences novatrices qui vont critiquer des procédés que le romancier lui-même a érigés en règle. De même, au plan stylistique, les discours normés ne sont là que pour servir d'écrin aux effets de rupture. Ils mettent ainsi en valeur les «faits de style», définis par M. Riffaterre comme l'irruption d'un élément imprévu à l'intérieur d'un contexte marqué par une certaine prévisibilitée ${ }^{35}$. Dans le Lancelot, l'invention narrative est portée par un style simple, sans recherche rhétorique particulière, qui valorise la sobriété et n'utilise guère les figures trop éclatantes. Le Tristan, lui, compense l'aspect répétitif des histoires qu'il raconte par une recherche qui porte sur les ornementations discursives du récit.

La pratique de la compilation ne fait que renforcer cet effet de contraste : le Tristan ne se donne même plus la peine de s'approprier, de naturaliser les séquences constituant l'ordinaire de la narration, il se contente de réutiliser ce qu'il trouve tout écrit chez d'autres. C'est le manque même de couleurs du tissu narratif simple du Tristan qui le rend susceptible d'accueillir ces morceaux venus d'autres univers textuels.

Dans ce déroulement prévisible, dans cet enchainement monotone, le Tristan va donc insérer deux types de morceaux qui s'imposeront comme sa marque propre: des passages inspirés par la lyrique, et des passages que l'on qualifiera de comiques. Exprimant des opinions opposées, en des styles qui le sont aussi, ces morceaux de bravoure aux tonalités différentes se rejoignent souvent par un même goût pour les antithèses, les proverbes, les métaphores concrètes. Bien loin de se nuire, ces deux types de discours se répondent et s'éclairent mutuellement. 


\section{NOTES}

1.E. Baumgartner, Essai d'interprétation d'un roman médiéval, Genève, Droz et Paris, Minard, 1975, p. 288.

2.D'où l'adoption, faute de mieux, du qualificatif « comique » comme caractéristique commode de ce registre. Pour une étude d'ensemble sur les aspects plaisants du roman médiéval, voir l'ouvrage de P. Ménard Le rire et le sourire dans le roman courtois en France au Moyen Age (1150-1250), Genève, Droz, 1969.

3.Sauf mention contraire comme ici, les citations renvoient aux tomes de l'édition menée sous la direction de P. Ménard : t. I, éd. P. Ménard, Genève, Droz, 1987 ; t. II, éd. M. L. Chênerie et T. Delcourt, 1990 ; t. III, éd. G. Roussineau, 1991 ; t. IV, éd. J.-C. Faucon, 1991, t. V éd. D. Lalande, 1992 ; t. VI, éd. E. Baumgartner et M. Szkilnilk, 1993. Le début du roman est cité d'après l'édition de R. L. Curtis, t. I, Munich, Max Hueber, 1963 ; t. II, Leiden, Brill, 1976 ; t. III, Cambridge, Brewer, 1985.

4.Voir le prologue de l'édition R. L. Curtis t. I, Munich, Max Hueber, 1963, p. 39 (où est annoncé le récit de «beles aventures et plesanz ») et l'épilogue du manuscrit BN fr 1628, transcrit par E. Baumgartner dans La Harpe et l'Epée, tradition et renouvellement dans le Tristan en prose, Paris, CDU-SEDES, 1990, p. 23.

5.Dire le vrai: l'adverbe « si » en français médiéval, Genève, Droz, 1985, p. 53.

6.Ibid., p. 115.

7.IV, pp. 149-162.

8.IV, p. 348 : « Aygrement parla Dynadans a ceste fois!».

9.Geoffroi de Vinsauf écrit par exemple cum loquimur de aliqua persona proba vel mala, vel hujus vel illius aetatis, debemus de persona proposita talia proponere quae convenianter attribuantur eidem personae, (Documentum de arte versificandi II, 3). Voir E. Faral, Les Arts Poétiques du XII et du XIII ${ }^{e}$ siècle, Paris, Champion, 1924, p. 310. Le Tristan en prose va cependant plus loin en attribuant à Marc des paroles indignes de sa qualité de roi. 10.IV, p. 278.

11.Ed. R.L. Curtis, t. III, p. 14.

12.Le Chevalier au Lion, Euvres complètes de Chrétien de Troyes, sous la direction de D. Poirion, Paris, Gallimard, 1994, v. 4098.

13.Dictionnaire des symboles, J. Chevalier et A. Gheerbrant, Paris, Laffon, 1969, pp. 798-799.

14.Le Roman de Renart, Ia, éd. J. Dufournet, Paris, Garnier-Flammarion, 1985, Vv. 1765-1818.

15.IV, p. 260

16.Le Roman de Renart, op. cit, br. Va, vv. 317-318.

17. Conception du langage comme un mécanisme binaire qui associe symbolisants (mots) et symbolisés (concepts) et renvoie à une vérité préexistante. Voir la distinction de Todorov entre signe et symbole dans « Introduction à la symbolique » Poétique 11, Seuil, 1972 et C. Reichler, La diabolie : la séduction, la renardie, l'écriture, éd. de Minuit, 1979.

18. Voir par exemple J. Scheidegger Le Roman de Renart ou le texte de la dérision, Genève, Droz, 1989, p. 19 : «Renart comme personnage entretient un rapport privilégié avec l'écriture de son roman ». 
19.II, pp. 353-363.

20.Voir pp. 103-120.

21.II, p. 111.

22.V, p. 132.

23.IV, p. 118

24.V, p. 217.

25.M-L. Ollier, « Proverbes et sentences : le discours d'autorité chez Chrétien de Troyes ", Revue des Sciences Humaines, 163, 1976, pp. 329-357.

26.Le rire et le sourire..., op. cit., p. 610.

27. Selon la terminologie d'E. Schulze-Busacker, Proverbes et expressions proverbiales dans la littérature narrative du Moyen Age français, Slatkine, 1985, p. 30 : « exploitation des possibilités lexicales et conceptuelles d'un proverbe pour une partie distincte d'un discours » (ici, le roman dans le roman du Vallet à la Cotte Maltaillée).

28.D'après E. Schulze-Busacker et $\mathrm{P}$. Ménard, on le trouve dans le Roman de Thèbes, dans le Brut, dans Le Chevalier de la Charrette, Le Chevalier au Lion et la Deuxième Continuation (Proverbes..., op. cit., pp. 246-248 ; Le rire et le sourire..., op. cit., pp. 612-613).

29.IV, p. 244

30.Ce proverbe, rapproché en note de « Li sens fault aucune foiz au besoing ", proverbe $\mathrm{n} 1117$ du recueil de J. Morawski (Proverbes français antérieurs au XV siècle, Paris, 1925) n'apparaît pas dans les romans arthuriens d'après les relevés d'E. Schulze-Busacker et de P. Ménard. En revanche, il nous paraît particulièrement proche d'expressions proverbiales présentes dans le Roman de Renart : « N'i a si sage ne foloit » (II, v. 431 ; VI, v. 380) ; « Mes en cest monde n'a si sage / Au chef de foiz n'aut a folage » (IV, vV. 31-32), « Mes sages hom sovent foloie» (IX, V. 594).

31. C'est ce que remarquait déjà E. Vinaver dans « Un chevalier errant à la recherche du sens du monde ", A la Recherche d'une Poétique médiévale, Paris, Nizet, 1970, pp. 163-177. 32.E. Baumgartner, Essai d'interprétation d'un roman médiéval, op. cit., p. 296.

33.V, p. 106

34.VI, pp. 359-364.

35.Essais de stylistique structurale, présentation et traduction de D. Delas, Flammarion, 1971, p. 57.

\section{AUTEUR}

\section{BÉNÉDICTE MILLAND-BOVE}

Université Paris-III Sorbonne nouvelle 